\title{
POSSIBILITIES FOR USING LIDAR AND PHOTOGRAMMETRIC DATA OBTAINED WITH AN UNMANNED AERIAL VEHICLE FOR LEVEE MONITORING
}

\author{
K. Bakuła ${ }^{\text {a, } * \text { W. Ostrowski }}{ }^{\text {a }}$, M. Szender ${ }^{\text {b }}$,W. Plutecki ${ }^{\text {b }}$, A. Salach ${ }^{\text {a }}$, K. Górski ${ }^{\text {a }}$ \\ ${ }^{a}$ Warsaw University of Technology, Faculty of Geodesy and Cartography, Department of Photogrammetry, Remote Sensing and \\ Spatial Information Systems, Warsaw, Poland - (k.bakula,w.ostrowski)@gik.pw.edu.pl, gor.konrad@gmail.com, adam.salach@o2.pl \\ ${ }^{\mathrm{b}}$ MSP Marcin Szender, Warsaw, Poland - (marcin.szender, wienczyslaw.plutecki)@uav.com.pl
}

\author{
Commission I, ICWG I/Vb
}

KEY WORDS: UAV, UAS, LiDAR, ultra-light, accuracy, DTM

\begin{abstract}
:
This paper presents the possibilities for using an unmanned aerial system for evaluation of the condition of levees. The unmanned aerial system is equipped with two types of sensor. One is an ultra-light laser scanner, integrated with a GNSS receiver and an INS system; the other sensor is a digital camera that acquires data with stereoscopic coverage. Sensors have been mounted on the multirotor, unmanned platform the Hawk Moth, constructed by MSP company. LiDAR data and images of levees the length of several hundred metres were acquired during testing of the platform. Flights were performed in several variants. Control points measured with the use of the GNSS technique were considered as reference data. The obtained results are presented in this paper; the methodology of processing the acquired LiDAR data, which increase in accuracy when low accuracy of the navigation systems occurs as a result of systematic errors, is also discussed. The Iterative Closest Point (ICP) algorithm, as well as measurements of control points, were used to georeference the LiDAR data. Final accuracy in the order of centimetres was obtained for generation of the digital terrain model. The final products of the proposed UAV data processing are digital elevation models, an orthophotomap and colour point clouds. The authors conclude that such a platform offers wide possibilities for low-budget flights to deliver the data, which may compete with typical direct surveying measurements performed during monitoring of such objects. However, the biggest advantage is the density and continuity of data, which allows for detection of changes in objects being monitored.
\end{abstract}

\section{INTRODUCTION}

Direct surveying measurements, such as tacheometry, levelling or GNSS measurements, are surveying techniques that are mostly applied to the inventory and monitoring of levees. Such measurements are characterized by their very high accuracy in the order of centimetres. However, they are expensive with respect to monitoring entire flood protection networks at the regional and national scales. Appointed administration units are responsible in particular countries for their implementation. Thousands of kilometres of levees of various categories, size and conditions should be permanently monitored. This requires acquisition of huge amounts of data. Due to budget limitations, it is sometimes not possible to perform these operations by means of surveying direct measurements within one year for all linear objects of hydrotechnical constructions or even less frequently. Remote and more efficient techniques of measurements such as photogrammetry and remote sensing could fulfil efficiency requirement for this task. They have been considerably developed with respect to their accuracy recently. They are now characterized by lower costs, higher effectiveness and shorter data acquisition time.

Within many programmes, huge sets of data, required for simulation of rising waters in order to generate flood hazard maps, are acquired; one of the most important techniques applied for this purpose is airborne laser scanning (ALS). The usefulness of the ALS technique is also important for monitoring dikes (i.e., Long et al., 2010; Bakuła, 2013). The advantage of these data is, first of all, their density and quasicontinuity of surface description, which - compared with direct measurements of levee cross-sections - is particularly clearly visible. Compared with other remote sensing techniques, ALS data are the source of the most accurate data acquisition technique concerning terrain elevation for large areas; they are characterized by the absolute accuracy of measurements within $10 \mathrm{~cm}$, with the relative accuracy of centimetres. They also allow shapes and relative elevations of a levee to be presented. The greatest difficulty related to this technology is the fact that repeated laser scanning flights are not performed frequently enough for measurements aimed at monitoring conditions of hydrotechnical structures. Terrestrial laser scanning (TLS), which has become a popular tool used by surveyors, may lead to accuracies similar to conventional measurement techniques, offering high data density at the same time (Flener et al., 2013; Tournadre et al., 2014). However, this technique is relatively economically inefficient and time-consuming. In order to scan both sides of a levee, the scanner must be placed on both sides (low efficiency) or on a levee, but this may result in occluded areas on escarpments. It may also be unsafe when there is a risk of flood in periods of rising water. Mobile mapping systems, mounted on wheel or floating platforms that register point clouds moving along such structures, may be the solution to this problem (e.g., Flener et al., 2013). However, many limitations exist in this case, from the lack of possibilities to move on the levee to the inability to register both sides of the levee at the same time (the water-facing side and the lowland side). Although measurements performed by means of platforms moving along levee are permitted in many places in periods with low flood risk, moving on waterlogged levees is strictly forbidden. This would make any measurements and monitoring

* Corresponding author 
of hydrotechnical structures impossible since waterlogged levees are soggy and moving on their surface may result in damaging or breaking the dikes. The disadvantage of the floating platform is the lack of possibilities to monitor the lowland side of the levee.

Unmanned aerial vehicles (UAV) or unmanned aerial systems (UAS) may be the solution to this problem. They have been utilized for several years in many projects related to implementation of high accuracy measurements within short time intervals, without the necessity of high financial inputs; also for monitoring of levees (Flener et al., 2013; Tournadre et al., 2014, 2015). Photogrammetry of linear features is connected with many georeference issues (Tournadre et al., 2015). However, the more difficult task is related to presentation of a terrain surface covered by vegetation. Recently, the rapid development of laser scanning technology has been observed; however, the acquisition of LiDAR data from unmanned aerial platforms has only become feasible within the last two to three years (Petrie, 2013). The possibilities for using an unmanned aerial system, equipped with an ultra-light laser scanner and a digital camera for evaluating conditions of levees are discussed in this paper. The LiDAR data processing methodology is also presented and the obtained results are discussed.

\section{EXPERIMENT}

The objective of the experiment was to test an unmanned aerial system, equipped with a laser scanner, to investigate the possibility of using it for the inventory and monitoring of levees and to develop a methodology that would allow the acquisition of data of satisfactory accuracy. According to legal regulations in many European countries concerning monitoring of earthwork flood prevention constructions the required accuracy of measurements must be within $10 \mathrm{~cm}$ (the most critical infrastructure). An important aspect of periodical monitoring of such constructions is not only to acquire geometric information, but also to recognize conditions within surrounding areas of the levees and to identify possible hazards caused by natural as well as anthropogenic factors. Therefore, within the experiments, not only typical, geometric products, such as the Digital Terrain Model (DTM) or the Digital Surface Model (DSM), were generated; colour point clouds and orthophotomaps allowing for recognition of surrounding areas were also produced. The tested platform was also equipped with a digital camera, allowing photogrammetric measurements to be taken additionally. However, during the tests, attention was focused on laser scanning and photographs were only used to generate an orthophotomap and to colourize the point cloud. In this section, a platform description, as well as the methodology allowing that platform to be utilized, is presented, followed by a description of the test sites and the acquired data.

\subsection{The platform}

Although a fixed-wing may be considered as a better platform for measuring large areas (Eisenbeiss and Sauerbier, 2011), legal limitations that force the flight within visual line of sight (VLOS) would not practically allow the advantages of such constructions over gyroplanes in the case of measurements of linear features. The sensitivity of sensors placed on the platform and the necessity to operate in a range of terrains suggest the selection of a platform that does not require a specially prepared airstrip and is easy for flying, particularly when low flight altitude is planned due to the accuracy of the utilized scanner. All these arguments were the basis for the decision to use a multirotor construction.
The modified Hawk Moth, which is a four rotor platform produced by the company MSP (Figure 1) was used for the project. Its maximum gross weight is $11.5 \mathrm{~kg}$ and the span of its propellers is above $80 \mathrm{~cm}$ (the parameters of the platform are listed in Table 1). The platform has been equipped with two photogrammetric sensors: the YellowScan Mapper laser scanner and the Sony $\alpha 6000$ digital camera with a $16 \mathrm{~mm}$ lens.

\begin{tabular}{|l|l|}
\hline Name & Hawk Moth \\
\hline Developer & MSP \\
\hline Empty weight & $5.9 \mathrm{~kg}$ \\
\hline Max. payload & Over $3 \mathrm{~kg}$ \\
\hline Max. gross weight & $11.5 \mathrm{~kg}$ \\
\hline Average cruise speed & $5 \mathrm{~m} / \mathrm{s}$ \\
\hline Max. cruise speed & $12.5 \mathrm{~m} / \mathrm{s}$ \\
\hline $\begin{array}{l}\text { Hovering time } \\
\text { (with 3 kg payload) }\end{array}$ & 15 minutes \\
\hline
\end{tabular}

Table 1. Selected parameters of the Hawk Moth platform

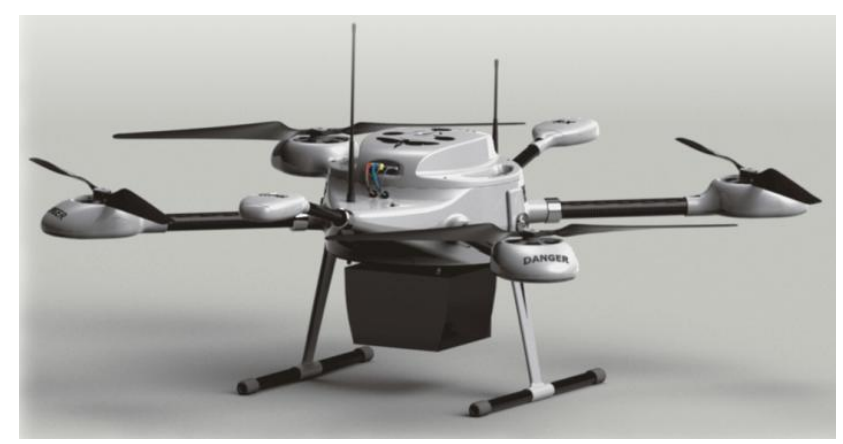

Figure 1. The Hawk Moth platform with the YellowScan scanner

The YellowScan laser scanner (Figure 2) is a laser scanner dedicated for use with light UAVs. It is characterized by lower weight (about $2.2 \mathrm{~kg}$ ) and lower price than higher class scanners such as Riegl VUX; in contrast to other budget solutions it is not based on the LiDAR Velodyne sensor (as in the case of Phoenix Aerial or RouteSense scanners); the applied sensor allows up to three returning echoes to be registered. The parameters of the LiDAR sensor are presented in Table 2. In addition, the scanner is also equipped with a navigation module with a double-frequency single-antenna GNSS receiver, allowing measurements in RTK and kinematic PPP modes to be performed, as well as an IMU sensor, which utilizes kinematic azimuth alignment.

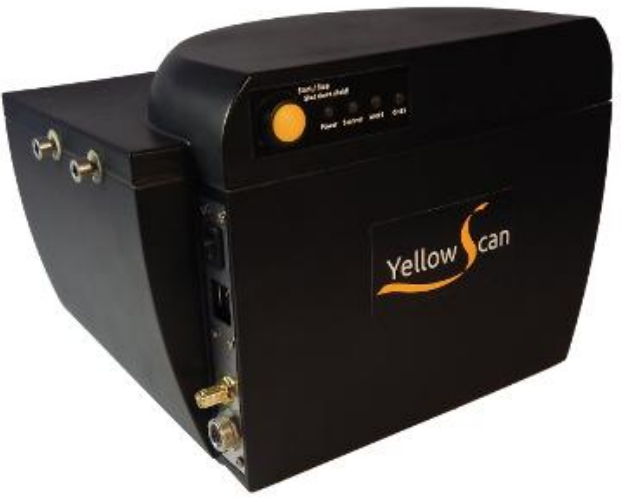

Figure 2. The YellowScan Mapper (Yellowscan. 2015) 


\begin{tabular}{|l|l|}
\hline Name & Mapper \\
\hline Developer & YellowScan \\
\hline Weight (with battery) & $2.2 \mathrm{~kg}$ \\
\hline Range & $100 \mathrm{~m}$ \\
\hline Echo registration & up to 3 \\
\hline Wavelength & $905 \mathrm{~nm}$ \\
\hline Divergence & $0.1 \times 0.8^{\circ}$ \\
\hline Scan angle & $+/-50^{\circ}$ \\
\hline Angular resolution & $0.125^{\circ}$ \\
\hline Frequency & $40 \mathrm{kHz}$ \\
\hline Accuracy & $10 \mathrm{~cm}$ \\
\hline
\end{tabular}

Table 2. Selected parameters of the LiDAR YellowScan Mapper

The theoretical scanning density may achieve up to 600 points per square metre; however, it depends on the altitude and speed of flight (Table 3). The performed experiments proved that the density is not constant across the scanned path. A considerable decrease in density is clearly visible (Figure 3 ) in its outer zones of strip, which may be caused by the use of few measuring sensors with overlapping field of view. The width of this part of the strip is equal to approx. one-sixth on each side.

\begin{tabular}{|c|c|c|c|c|c|c|c|c|c|c|c|c|}
\hline & \multicolumn{12}{|c|}{ Altitude [m] } \\
\hline \multirow{10}{*}{ Speed $[\mathrm{m} / \mathrm{s}]$} & & 10 & 15 & 20 & 25 & 30 & 35 & 40 & 45 & 50 & 55 & 60 \\
\hline & 2.8 & 600 & 400 & 300 & 240 & 200 & 173 & 152 & 135 & 121 & 110 & 101 \\
\hline & 4.2 & 400 & 267 & 200 & 160 & 133 & 116 & 101 & 90 & 81 & 73 & 67 \\
\hline & 5.6 & 300 & 200 & 150 & 120 & 100 & 87 & 76 & 67 & 61 & 55 & 50 \\
\hline & 6.9 & 240 & 160 & 120 & 96 & 80 & 69 & 61 & 54 & 48 & 44 & 40 \\
\hline & 8.3 & 200 & 133 & 100 & 80 & 67 & 58 & 51 & 45 & 40 & 37 & 34 \\
\hline & 9.7 & 171 & 114 & 86 & 69 & 57 & 50 & 43 & 38 & 35 & 31 & 29 \\
\hline & 11.1 & 150 & 100 & 75 & 60 & 50 & 43 & 38 & 34 & 30 & 27 & 25 \\
\hline & 12.5 & 133 & 89 & 67 & 53 & 44 & 39 & 34 & 30 & 27 & 24 & 22 \\
\hline & 13.9 & 120 & 80 & 60 & 48 & 40 & 35 & 30 & 27 & 24 & 22 & 20 \\
\hline
\end{tabular}

Table 3. Theoretical emitted pulse density with respect to speed and altitude (based on Yellowscan, 2015)

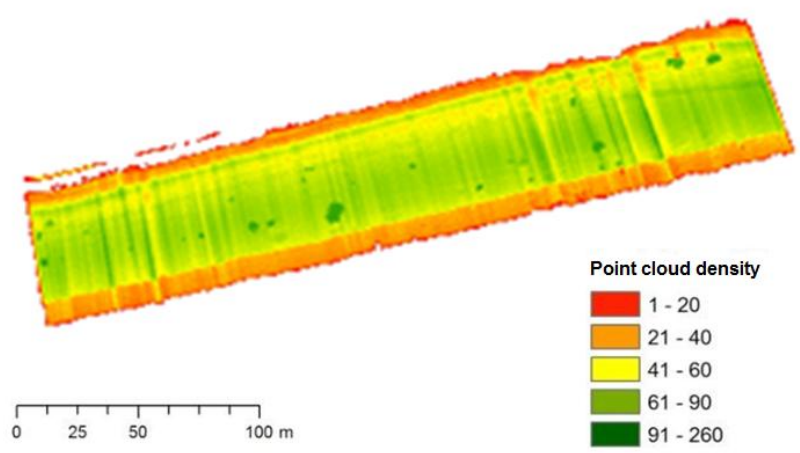

Figure 3. The point cloud density for a single scanned path from an ultra-light scanner (flight altitude 30 AGL)

The other photogrammetric sensor mounted on the platform is a Sony $\alpha 6000$ digital camera with the array APS-C $(23.2 \times 15.4 \mathrm{~mm})$ with a resolution of $24.3 \mathrm{Mpix}$ (dimensions of a photograph are $6000 \times 4000$ pixels). It allows the capture of photographs with full resolution in a two-second cycle. A wideangle lens with a focal length of $\mathrm{f}=16 \mathrm{~mm}$ was applied. The platform is also equipped with an independent navigation system with automatic pilot, allowing planned missions to be performed, using the determined path, altitude and flight speed and with shutter release in specified locations.

\subsection{The proposed methodology of data acquisition and processing}

The efficient monitoring of levees requires implementation of measurements and recognition of conditions of the linear structure and also of its direct neighbourhood. The width of such a scan strip has been specified by hydraulic engineers as $20 \mathrm{~m}$ (as the minimum) on both sides of the levee axis. Two approaches to data acquisition with the use of the tested platform were proposed. They assume that measurements of the area will be performed in one or two strips. The defined parameters of the width of scanning footprint indicate that a single flight should be sufficient in the majority of cases; however, in some case it might cause the creation of occluded areas.

The first case was the least complicated. The flight should be performed in such a way that the strip axis is located similarly to the axis of the linear feature and that a footprint has sufficient width. The footprint depends on the flight altitude. The second variant requires that appropriate overlapping between parallel scanning strips. A 75\% overlap between strips was assumed. This allows the elimination of data for the outer one-sixth scan footprint, at the time of data processing, due to the rapid decrease in the density. Data for this part might be considered as data of insufficient quality, mainly caused by the increasing influence of random errors of determination of the flight trajectory on the accuracy of measurements when the scanning angle increases.

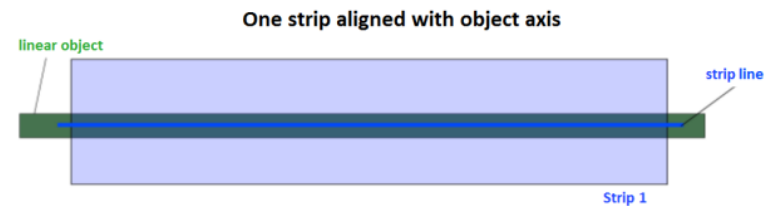

Two parallel strips

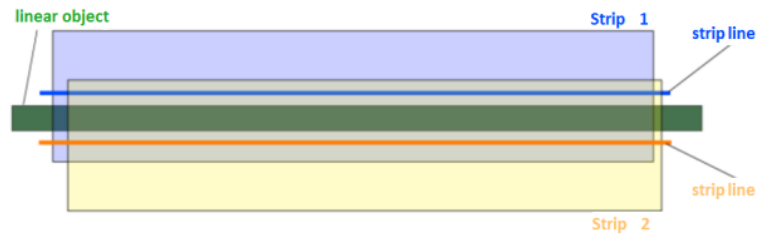

Figure 4. The applied variants of flights along levees: with one strip and with two strips of photographs

Considering the simultaneous operations of the camera and the laser scanner, the flight parameters (the flight altitude and the platform velocity) must be selected for both sensors. Determination of the optimum parameters of the flight altitude and speed required consideration of several limitations resulting from the requirements of equipment. One of the most important conditions concerns the maximization of the flight speed in order to increase the IMU accuracy, in accordance with the manufacturer's guidelines. However, the flight speed is also limited by the cycle of operations of the camera, which simultaneously registers digital photographs (at least every two seconds in this case) and the assumed forward overlapping, which should not be less than $60 \%$. Selection of the appropriate flight altitude is limited by three criteria. Due to the requirements concerning the emergency parachute, the flight altitude may not be lower than $30 \mathrm{~m}$ AGL additionally. 
Therefore, it is the preferred flight altitude, since - from the data acquisition perspective - lower altitude allows better accuracy of data acquisition with the use of the scanner (Yellowscan, 2015) to be achieved. The flight altitude also influences the ALS data point density, which is particularly important for the accuracy of acquired data. The GSD value of the acquired digital photographs varies proportionally to the flight altitude. The relationships between over-mentioned issues related to both sensors are presented in Figure 5.

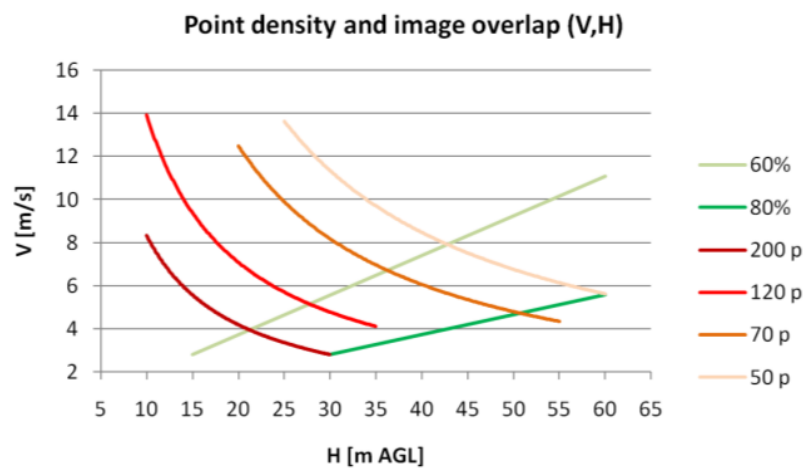

Figure 5. A diagram with optimizing flight parameters for both sensors

Additionally, a methodology for more complex and curved levees should be developed, which must ensure sufficient overlap, allowing for its implementation in the software used for the platform flight planning. The axes of the planned strips must be straight sections and no turns are allowed during the data registration in a strip. Every apex of the dike, which requires modification of the flight directory, also requires a given strip to be completed and the new strip to be commenced, adjusted to the new axis of the object. The key issue is the correct connection of strips, which may be performed only when the appropriate overlap of strips at their edges is performed. The flight should be planned with a sufficient buffer at the end of each section. This buffer must be sufficiently long to ensure the double overlap of the apex after turning and beginning the registration in the new strip. The double overlap and the ground control, densified in locations of apexes, ensure the accurate connection of strips. This situation is presented in the diagram below (Figure 6).

The first operation at the stage of data selection for further processing was to separate parts of the trajectory on the scanner software, for which las files were to be generated. Data collection was commenced before the start and was completed after landing and turning off the registration. During separation of strips the required separation from the beginning of the turn, at the end of the strip, was maintained. The separation into strip is necessary since the proposed methodology of the relative orientation, described further in the paper, assumes that particular strips are rigid bodies. Separation, maintained from the turning point, is required due to the trajectory adjustment model, applied in the YellowScan scanner.

The next operation of laser scanner data processing was to consider offsets and the scanner calibration results, as well as postprocessing of GNSS observations, using the PPP method, which is the obligatory operation if the RTK was not used at the data collection stage.

The next stage of ALS data processing is the relative orientation of the scanning strips. It is a conventional operation in the case when areas are scanned in neighbouring strips, within entire blocks. This results from the existence of systematic errors, varying in time and connected with the accuracy of the flight trajectory, determined by the GNSS and IMU systems (Kager, 2004). This effect was also observed in the case of data acquired from the ultra-light laser scanner. In the case of the ALS technique, such works are usually performed by means of the matching of detected planes (Filinand Vosselman, 2004; Pfeifer et al, 2005).

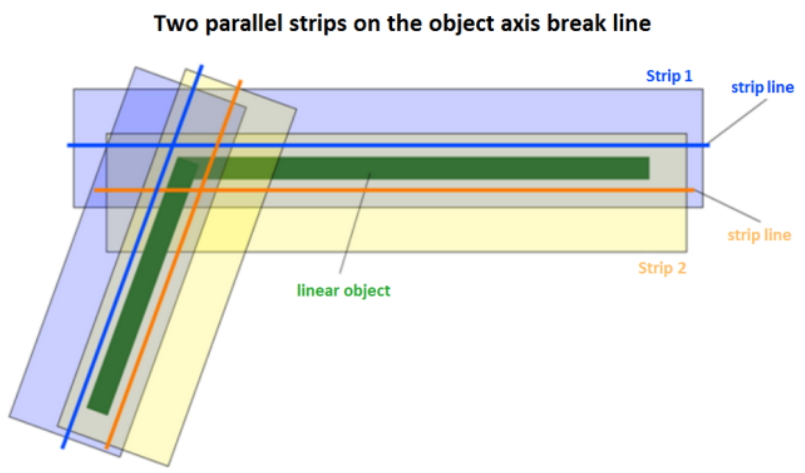

Figure 6. The variant of the flight along levees when the linear feature is turning

In the case of data of a small field range, a sufficient number of planes might not be found (connected with anthropogenic objects). Therefore, the described methodology proposes to utilize the ICP (Iterative Closest Points) algorithm, implemented in many software tools. The ICP algorithm searches for the transformation matrix, which includes rotations and translation between two point clouds in the common coordinate system. The concept consists of searching for the nearest pairs of points from two data sets and then minimizing the distance between them, in the iterative adjustment process (Eggert and Daylot, 2012). Such an approach can be performed with respect to ALS strip adjustments (Glira et al, 2015). One point cloud is considered as the reference cloud and the second cloud is matched to the first one. Since the successive strips were only partially overlapped, it should be considered that nearest pairs of points detected by the algorithm are not the pairs of "corresponding points". Therefore, it is necessary to determine the area of overlap between neighbouring scans, which may be determined in two ways:

- determination of the defined percentage of overlap (predefined-overlap),

- creation of a vector layer of the common part, including the area of overlap between scans and adjustment of the clipped point clouds (footprints-intersection)

The predefined-overlap-based method consists of the direct utilization of the ICP algorithm on raw point clouds. The algorithm is performed for full ranges of point clouds with the defined overlap between strips (as a percentage). It is the statistical approach when the overlap is arbitrarily defined. The footprints-intersection-based method utilizes the transformation matrix, generated on the basis of the common part of both scans only, determined as the intersection of footprints of neighbouring scanning strips; it is then used to match the entire point cloud into the reference scan strip.

The performed tests also included an analysis of the possibility of considering orientation for entire point clouds, as well as for the "ground" class only, testing the possibility of the influence of scanning land cover elements from various directions on the 
result of the relative orientation (georeferencing) of the point cloud. The first two variants utilized all points of the cloud; this is in accordance with recommendations concerning ALS data processing (Toth, 2008). In the case of these approaches one strip was automatically transformed to the other. However, in the case of variable overlap this method might generate errors of relative orientation. For the third and fourth variants, when filtration of each point cloud was performed before the adjustment, only points from the "ground" class were utilized in the ICP algorithm. Finally, four variants - two for the first method and two for the second method - were tested:

1. the predefined-overlap-based method (all points),

2. the footprints-intersection-based method (all points),

3. the predefined-overlap-based method (ground),

4. the footprints-intersection-based method (ground).

Filtering of ALS data from the ultra-light scanner is not a simple task, due to the small diversification of the terrain topography, considerable slopes on levees (even up to 60\%) and vertical concrete walls and other hydrotechnical structures, which often occur. The considerable density of the point cloud, with redundant observations that support the scanner accuracy, also does not facilitate the process of filtering. It was decided to utilize the algorithm of filtration of the "ground" class points using the adaptive TIN method (Axelsson, 2000). Filtering was performed using the LASTools software.

The final stage of airborne laser scanning data in the presented methodology uses the integration of scanning and photographs. The oriented point cloud was colourized based on the orthophotomap from aerial photographs. Although this procedure was not necessary, it improved interpretation possibilities of the point cloud. The simplest method of colourizing the point cloud is the use of an orthophotomap from digital photographs; it has been implemented in the LASTools package. It is possible to directly apply the georeference in order to colourize the cloud with projected photographs; however, it was decided to perform aerial triangulation for simultaneously acquired photographs with the use of conventional ground control points and then to orthogonalize oriented photographs on the DTM from ALS, which leads to better information and reduces the generation time of point clouds from digital image-matching.

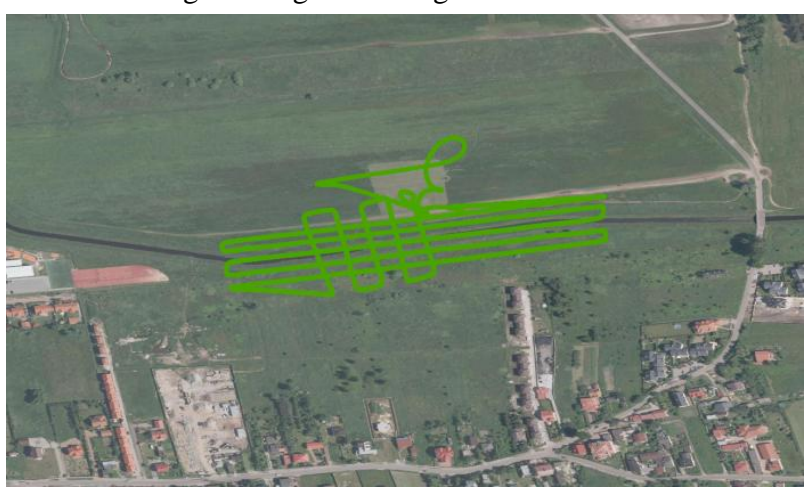

Figure 7 . The example trajectory of the test flight

\subsection{The test site and the test flight}

The test site is a section of the Długa River in Warsaw (Białołęka district) with a length of approximately $400 \mathrm{~m}$. The river bed of the width of approx. $5 \mathrm{~m}$ is protected on both sides with levees of the relative height of $2 \mathrm{~m}$. The trajectory of the test flight consisted of five strips along flight and five strips across flight (Figure 7). Measurements were performed at the altitude $\mathrm{h}=30 \mathrm{~m}$ AGL (above the ground level) with the mean velocity of $5 \mathrm{~m} / \mathrm{s}$. Two strips along levee were selected for data processing by means of GIS software extensions.

Within the test site, 121 spot heights were measured by means of the RTK technology, using the local reference network (TPI NETPro). Elevations were calculated for the Kronsztadt' 86 system, using the point of the control network (normal elevations). Coordinates of 16 ground control points, marked in the field, were also measured for further processing of acquired photogrammetric data (as control and check points).

\section{RESULTS}

Preparation of data acquired from the ultra-light scanner (import, calibration and georeference postprocessing) described above was performed for the discussed test site. In this section the results of relative orientation of the strips acquired from the light scanner, as well as the accuracy and layout of obtained results, used for monitoring of levee conditions, are presented.

Due to the required accuracy of the final product, similar to the accuracy of the scanner, selection of the relative orientation method in the case where more than one strip exists was important during the described works. The accuracy of matching point clouds using the given method was determined by the difference between the digital terrain model from the matched point cloud and the digital terrain model from the reference point cloud. Table 4 presents the results of matching neighbouring scans using different variants of the ICP algorithm, based on two methods (the predefined-overlap-based and the footprints-intersection-based method) with determination of transformation parameters on the basis of matching the entire point cloud or only the "ground" points. It shows that filtration preceding the ICP processing of the point cloud and the use of only the "ground" points does not influence the improvements of the results of matching. Both the predefined-overlap-based (ground) method, as well as the footprints-intersection-based (ground) method did not significantly improve the accuracy of matching of neighbouring scans. The highest accuracy was obtained for the ICP footprints-intersection-based method - the expected matching accuracy equalled approx. $5 \mathrm{~cm}$. It is worth noticing that differences between the results are not high and such comparisons may require further testing for the bigger number of longer linear features.

\begin{tabular}{|c|c|c|c|c|}
\hline \multirow{2}{*}{} & \multicolumn{4}{|c|}{ ICP Method } \\
\cline { 2 - 5 } & $\begin{array}{c}\text { ICP } \\
\text { predefined- } \\
\text { overlap- } \\
\text { based } \\
\text { (all points) }\end{array}$ & $\begin{array}{c}\text { ICP } \\
\text { footprints- } \\
\text { intersection } \\
\text {-based } \\
\text { (all points) }\end{array}$ & $\begin{array}{c}\text { ICP } \\
\text { predefined- } \\
\text { overlap- } \\
\text { based } \\
\text { (ground) }\end{array}$ & $\begin{array}{c}\text { ICP } \\
\text { footprints- } \\
\text { intersection } \\
\text {-based } \\
\text { (ground) }\end{array}$ \\
\hline $\begin{array}{c}\text { Mean } \\
\text { value }\end{array}$ & $0.020 \mathrm{~m}$ & $\mathbf{0 . 0 1 8 ~ \mathbf { ~ m }}$ & $0.008 \mathrm{~m}$ & $0.009 \mathrm{~m}$ \\
\hline $\begin{array}{c}\text { Standard } \\
\text { deviation }\end{array}$ & $0.068 \mathrm{~m}$ & $\mathbf{0 . 0 4 8 ~ \mathbf { ~ m }}$ & $0.058 \mathrm{~m}$ & $0.053 \mathrm{~m}$ \\
\hline
\end{tabular}

Table 4. Results of matching neighbouring scans, performed by means of different ICP methods 
A) Absolute cloud to cloud distance before strip adjustment

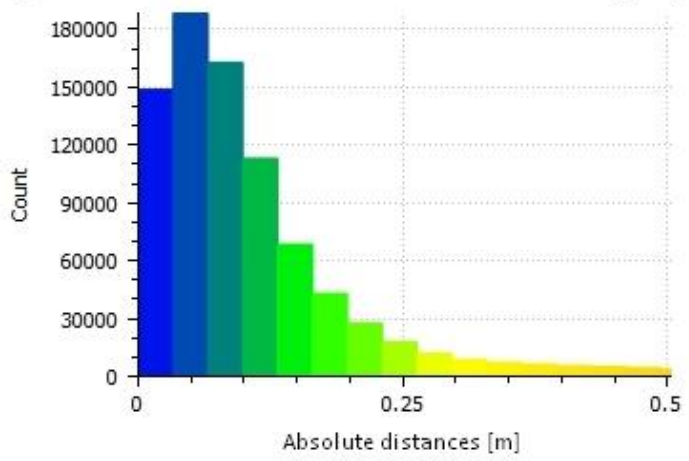

B) Absolute cloud to cloud distance after strip adjustment

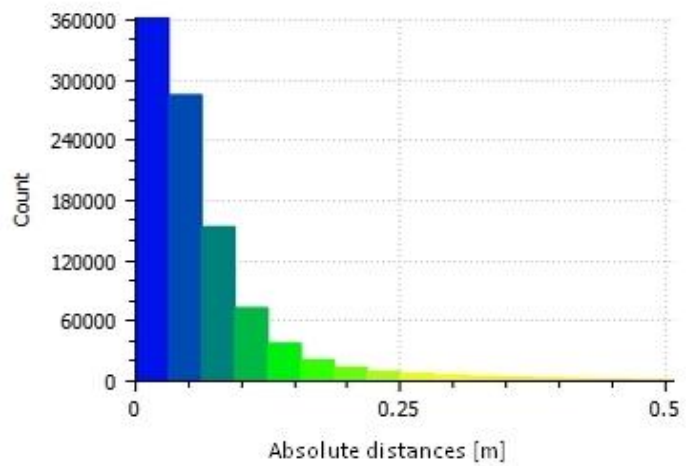

Figure 8. A histogram presenting the absolute distance between point clouds from neighbouring strips:

A) before strip matching B) after strip matching

However, for the selected best method the histograms below present the absolute distance between point clouds from neighbouring strips: before (Figure 8a) and after (Figure 8b) matching of strips. The histogram in Figure 8a shows that the majority of corresponding, calculated distances between points fall in the interval reaching up to $20 \mathrm{~cm}$. After matching the strips, a decrease in the absolute distance of point clouds is clearly visible - the majority of points fall in the interval $0-10 \mathrm{~cm}$. For comparison, the number of points in the interval between 0 and $5 \mathrm{~cm}$ was increased more than twice as a result of the use of the ICP algorithm.

The relatively oriented two strips (or elimination of this stage in the case of one strip) were the subject of orientation of the block of scans connected with measurements of points in the intensity image. Georeference was performed by "reference controls" (of the size $1 \times 1 \mathrm{~m})$, which were scanned during the flight; coordinates of their centres were determined by the RTK method during field measurements (equivalent to the photogrammetric control).

Next, filtration of generated point clouds was performed in order to generate appropriate digital elevation models. The basic objective was to separate points of the "ground" class. The effect of filtration is visible in Figure 9, where the digital terrain model (after filtration) and the digital surface model, generated from the entire point cloud, are visible. Data prepared in this way allowed the generation of raster elevation models (DTM and DSM). They were automatically generated based on appropriate groups of points created as a result of filtration. Points from the "ground" class were used to create the DTM and points from the first return of the laser beam were used to create the DSM. Generated terrain models were the subject of the accuracy analysis; in the case of the single strip, along the dike's line and in the case of the two strips, on both sides. Table 5 presents the mean value of the difference and its standard deviation for 121 spot heights. The results show a small vertical systematic error. In both cases the standard deviation proves that the assumed accuracy of $10 \mathrm{~cm}$ was achieved.

\begin{tabular}{|c|c|c|}
\hline & $\begin{array}{c}\text { Mean value of height } \\
\text { difference }-\mathrm{dH}\end{array}$ & $\begin{array}{c}\text { Standard } \\
\text { deviation } \sigma\end{array}$ \\
\hline $\begin{array}{c}\text { Two parallel } \\
\text { strips }\end{array}$ & $-2.1 \mathrm{~cm}$ & $7.2 \mathrm{~cm}$ \\
\hline Single strip & $-1.4 \mathrm{~cm}$ & $8.6 \mathrm{~cm}$ \\
\hline
\end{tabular}

Table 5. Values for height differences between the DTM from laser scanning and GNSS-RTK measurements
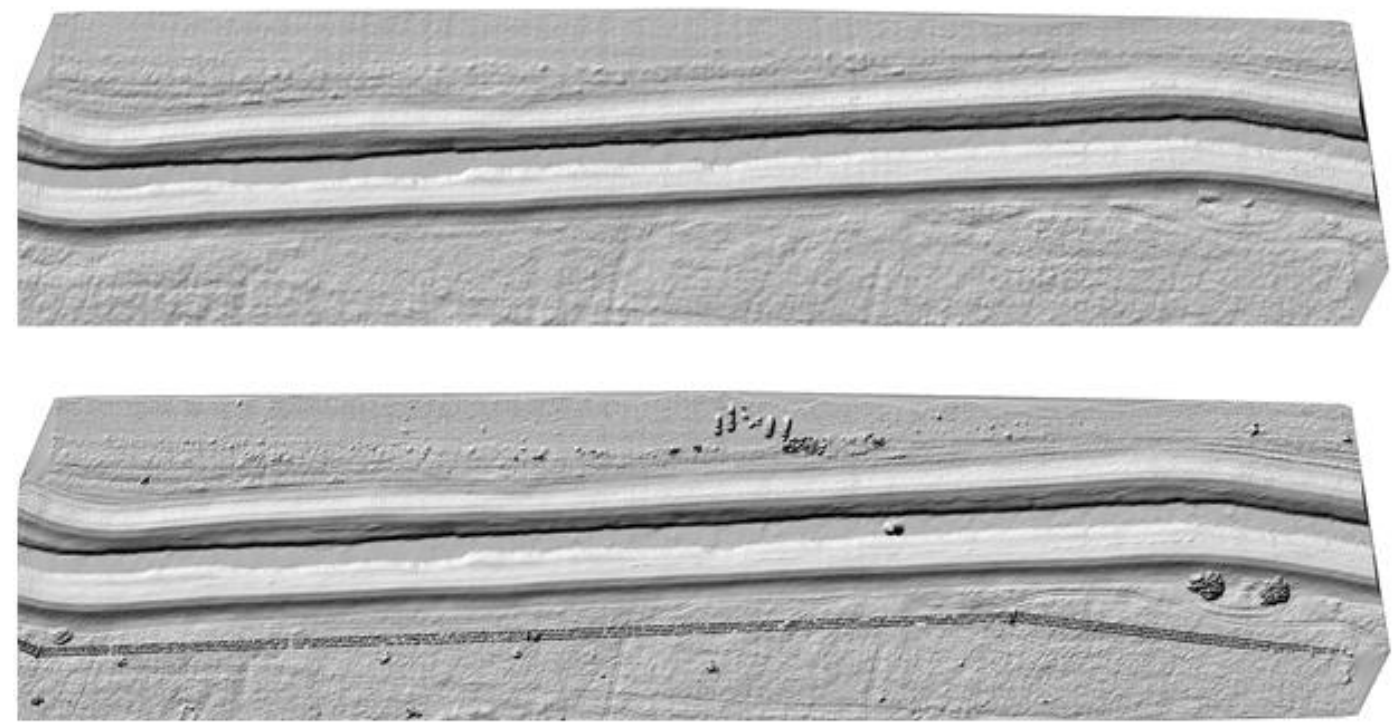

Figure 9. The Digital Terrain Model and the Digital Surface Model 


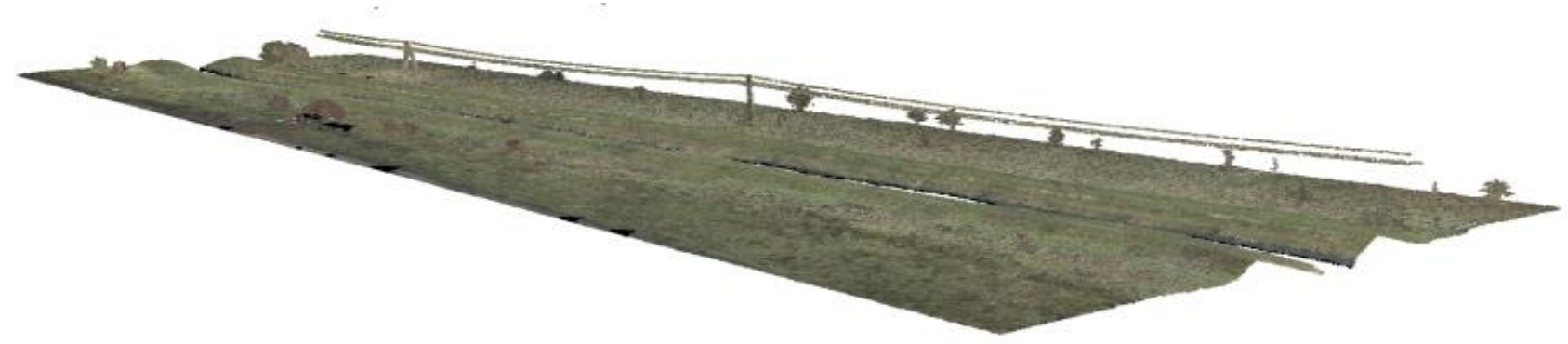

Figure 10. A colour point cloud from an ultra-light scanner, acquired from the UAV and coloured using photographs acquired from low altitude

The oriented point cloud was finally colourized by assigning every pixel with the RGB colour from the orthophotomap to the given point from the point cloud using corresponding coordinates. Colourizing was performed with the use of the orthophotomap from 71 photographs in two parallel strips, which were oriented using six ground control points (located in pairs, every 125 metres), which was evaluated with 10 check points. The RMS error values for the check points were: RMS $X=3.7 \mathrm{~cm}$, RMS $Y=5.1 \mathrm{~cm}$ and RMS $Z=6.3 \mathrm{~cm}$. The results of colourizing the point cloud are presented in Figure 10

\section{DISCUSSION}

The discussed platform and mounted sensors seem to be an appropriate configuration for monitoring of linear features, such as levees. Earlier studies concerning monitoring of dikes with the use of UAV data were focused on the use of low-altitude images (Tournadre et al., 2014, 2015). The presented platform is equipped with a laser scanner and digital camera; however, considering the objectives of acquisition of digital photographs, they were not the basic source of 3D information.

The applied scanner enabled an assumed accuracy of below $10 \mathrm{~cm}$ to be achieved. This was the result of many redundant observations as well as a scan relative orientation, which was carefully performed (in the case of two strips). Despite the recent, rapid development of technology of light laser scanners, it seems that in the near future it will not be possible to considerably improve acquired accuracy using inexpensive devices. The results from research carried out by Glennie et al. (2016) indicate that accuracies achieved in the course of the described studies are close to the measuring possibilities of applied LiDAR sensors. It is also worth noticing that those accuracies are sufficient from the perspective of autonomous vehicles. The solution that guarantees more accurate measurements is photogrammetry. Tournadre et al. (2014), as a result of UAV data image matching, obtain a DTM with an accuracy of $4 \mathrm{~cm}$; this accuracy was estimated based on TLS measurements. In theory (Cramer, 2016), it is possible to reach accuracy of even a sub-centimetre level. However, in the case of levee monitoring using photographs, key limitations are related to the vegetation (in particular grasses) covering such features.

When one decimetre accuracy is satisfactory, Flener et al. (2013) point out that acquisition of UAV photogrammetric data is sufficient. However, when better accuracy is required, he recommends the additional use of a mobile laser scanning technique. The accuracy of airborne laser scanning, which is often performed within regional projects with high budgets, is equal to approx. $10 \mathrm{~cm}$. However, levees must be monitored in cycles and the land cover is often insufficient to reliably describe the geometry of hydrotechnical structures using photogrammetric methods. This is the basic advantage of the ALS-based DTM over photogrammetric data. As experiments have shown (Esposito et al., 2014) the advantage of the LiDAR technique in areas covered by vegetation may also be noticed in the case of light laser scanners mounted on UAV platforms. Another advantage of using laser scanners is the data access time. Low-altitude UAV data may be quickly organized, which is a less expensive solution than the use of an aeroplane with the ALS system. Although the workflow presented in this paper is relatively wide, it is worth noticing that it requires less time than methods based on dense image matching and accessible technological solutions using the RTK method allow online access to acquired data (Kaul et al., 2015).

\section{CONCLUSIONS AND FUTURE WORK}

Independently of the applied sensors, UAV platforms seem to be the best available solution for monitoring flood levees, which allow the measurement of sections of more than 10 kilometres within one day; they are economically superior to the direct measurements technique as well as terrestrial scanning and they ensure that the required accuracy is achieved. The advantage of the UAV platform equipped with a laser scanner is, first of all, the non-invasive method of data acquisition. The unmanned aerial system is more mobile, since measurements may be quickly performed on both sides of a river and, in the case of a narrow river - even at the same time. The scanning range of the unmanned platform moving above a linear object is much wider than measurements that could be taken from the earth's surface, with the use of mobile platforms located beside the levees (surrounding roads, scanning from floating platforms). This may be particularly important in the case of emergency situation when it is difficult to access levees.

The performed experiments prove the high potential of ALS data acquired from UAV platforms that deliver products of several centimetres' accuracy, comparable with the direct measurement techniques. However, this technique is still relatively expensive. Its use is justified by the possible penetration of the vegetation cover, which usually covers the flood levees and influences the limitations on measuring their geometry using cheaper and accurate photogrammetric techniques. The market for ultra-light scanners dedicated for UAV platforms is still in its infancy; therefore, it is possible that the development of such sensors may undergo dynamic and significant change in the future. Numerous problems related to the current scanners exist, but the presented experiments confirm that many of them may be solved by means of cheaper 
tools, being the components of ALS data processing software, as well as of GIS applications.

A series of research topics still requires verification, such as the integration of LiDAR data and point clouds obtained from dense image matching in order to acquire reliable 3D information, or analysis of the influence of various laser scanners, including assessment of the influence of recording several returns by ultra-light scanners. Future research planned by the authors will concern the use of the proposed methodology of processing the data acquired from the light laser scanner for long linear features of many kilometres. This methodology will be verified for a range of test sites within financed studies, which will use LiDAR point cloud from UAVs together with historical aerial and satellite data, as well as direct measurements, for assessing the levees, creating a system for monitoring the conditions of flood levees (SAFEDAM).

\section{ACKNOWLEDGEMENTS}

This study was performed with the cooperation of the Warsaw University of Technology, the Faculty of Geodesy and Cartography with the company MSP, within the frames of delivery and commissioning of the unmanned platform, equipped with laser scanner and digital camera, for the benefit of the Institute of Meteorology and Water Management National Research Institute.

\section{REFERENCES}

Axelsson, P. 2000. DEM generation from Laser Scanner Data using Adaptive TIN Models. International Archives of Photogrammetry and Remote Sensing, XXXIII, Part B4, Amsterdam 2000.

Bakula, K. 2013. The Effective application of geospatial data in flood hazard and risk map creation. International Multidisciplinary Scientific GeoConference: SGEM: Surveying Geology \& mining Ecology Management, 381.

Cramer, M. 2016. UAS photogrammetry for high precise point positioning of linear objects. EuroCOW 2016, the European Calibration and Orientation Workshop (presentation), 10-12 February 2016, Lausanne, Switzerland.

Eggert, D., Dalyot, S., 2012. Octree-based simd strategy for ICP registration and alignment od 3D point clouds. ISPRS Annals of the Photogrammetry, Remote Sensing and Spatial Information Sciences, Volume I-3, 2012 XXII ISPRS Congress, Melbourne, Australia, 105-110.

Eisenbeiss, H., \& Sauerbier, M. 2011. Investigation of UAV systems and flight modes for photogrammetric applications. Photogrammetric Record, 26(136), 400-421.

Esposito, S., Mura, M., Fallavollita, P., Balsi, M., Chirici, G., Oradini, A., \& Marchetti, M. 2014. Performance evaluation of lightweight LiDAR for UAV applications. International Geoscience and Remote Sensing Symposium (IGARSS), 792795.

Flener, C., Vaaja, M., Jaakkola, A., Krooks, A., Kaartinen, H., Kukko, A., Kasvi, E., Hyyppä, H., Hyyppä, J. and Alho, P., 2013. Seamless mapping of river channels at high resolution using mobile LiDAR and UAV-photography. Remote Sensing, 5(12), pp. 6382-6407.
Filin, S. and Vosselman, G., 2004. Adjustment of airborne laseraltimetry strips. In: International Archives of Photogrammetry and Remote Sensing, XXXV, B3, Istanbul, Turkey, p. 5.

Glennie, C. L., Kusari, A., \& Facchin, A., 2016. Calibration and stability analysis of the VLP-16 laser scanner. ISPRS International Archives of the Photogrammetry, Remote Sensing and Spatial Information Sciences, XL(1), 10-12.

Glira, P., Pfeifer, N., Christian, B., \& Camillo, R., 2015. A Correspondence Framework for ALS Strip Adjustments based on Variants of the ICP Algorithm. Photogrammetrie, Fernerkundung, Geoinformation, 2015.4, 275-289.

Kager, H., 2004. Discrepancies between overlapping laser scanner strips-simultaneous fitting of aerial laser scanner strips. International Archives of Photogrammetry, Remote Sensing and Spatial Information Sciences, 35(B1), 555-560.

Kaul, L., Zlot, R., \& Bosse, M. 2015. Continuous-Time ThreeDimensional Mapping for Micro Aerial Vehicles with a Passively Actuated Rotating Laser Scanner. Journal of Field Robotics, 33(1), 103-1032.

Lichti, D. \& Skaloud, J., 2010. Registration and Calibration. Vosselman, G.; Maas, H.-G. (Eds.). Airborne and Terrestrial Laser Scanning, 1-42.

Long, G., Mawdesley, M. J., Smith, M., \& Taha, A., 2010. Simulation of airborne LiDAR for the assessment of its role in infrastructure asset monitoring. Proceedings of the international Conference on computing in civil and building engineering, Tizani W. (ed). Nottingham University Press, Nottingham, UK.

Petrie, G., 2013. Current developments in airborne laser scanners suitable for use on lightweight UAVs: Progress is being made!. GeoInformatics, 16(8), 16-22.

Pfeifer, N., Elberink, S. O., \& Filin, S., 2005. Automatic tie elements detection for laser scanner strip adjustment. International Archives of Photogrammetry and Remote Sensing, 36(3/W3), 1682-1750.

Toth,, C.K., 2008. Strip Adjustment and Registration. - Shan, j.\&toth, c.k. (eds.): Topographic Laser Ranging and Scanning: Principles and Processing. - CRC Press.

Tournadre, V., Pierrot-Deseilligny, M., \& Faure, P. H., 2014. UAV photogrammetry to monitor dykes - calibration and comparison to terrestrial LiDAR. International Archives of Photogrammetry, Remote Sensing and Spatial Information Sciences, XL-3/W1, 143-148.

Tournadre, V., Pierrot-Deseilligny, M., \& Faure, P. H. (2015). UAV linear photogrammetry. International Archives of Photogrammetry, Remote Sensing and Spatial Information Sciences, 40(3), 327.

YellowScan 2015. User Manual version 1.5. 